\title{
Whatever the cost? Information integration in memory-based inferences depends on cognitive effort
}

\author{
Benjamin E. Hilbig • Martha Michalkiewicz • \\ Marta Castela • Rüdiger F. Pohl • Edgar Erdfelder
}

Published online: 12 December 2014

(C) Psychonomic Society, Inc. 2014

\begin{abstract}
One of the most prominent models of probabilistic inferences from memory is the simple recognition heuristic $(\mathrm{RH})$. The RH theory assumes that judgments are based on recognition in isolation, such that other information is ignored. However, some prior research has shown that available knowledge is not generally ignored. In line with the notion of adaptive strategy selection - and, thus, a trade-off between accuracy and effort-we hypothesized that information integration crucially depends on how easily accessible information beyond recognition is, how much confidence decision makers have in this information, and how (cognitively) costly it is to acquire it. In three experiments, we thus manipulated (a) the availability of information beyond recognition, (b) the subjective usefulness of this information, and (c) the cognitive costs associated with acquiring this information. In line with the predictions, we found that RH use decreased substantially, the more easily and confidently information beyond recognition could be integrated, and increased substantially with increasing cognitive costs.
\end{abstract}

Keywords Adaptive decision making · Information integration $\cdot$ Fast and frugal heuristics $\cdot$ Recognition heuristic $\cdot$ Multinomial processing tree models

Often enough, people must make judgments and decisions under uncertainty, aiming to infer or predict some distal criterion based on information that is only probabilistically related to this criterion. Doctors regularly infer a specific illness based on symptoms, brokers may need to predict stock prices from

B. E. Hilbig $(\square)$

Cognitive Psychology Lab, Department of Psychology, University of

Koblenz-Landau, Fortstraße 7, 76829 Landau, Germany

e-mail: hilbig@uni-landau.de

M. Michalkiewicz $\cdot$ M. Castela $\cdot$ R. F. Pohl $\cdot$ E. Erdfelder

University of Mannheim, Mannheim, Germany information such as company revenues, human resource managers attempt to predict job performance from test scores and the like, and consumers could try to infer how healthy food options are based on nutrition information. That is, distal criteria must be inferred or predicted from proximal information that is probabilistically related to the criterion (Brunswik, 1955; Gigerenzer et al., 1991). Given the manifold real-life examples of probabilistic inferences, it is all the more important to understand how these types of judgments are formed. Correspondingly, much research efforts have been exerted to clarify the cognitive processes behind judgments and decision making (Weber \& Johnson, 2009).

Among the different approaches to studying how people form judgments, the adaptive toolbox framework (Gigerenzer, 2001; Gigerenzer \& Selten, 2001) has gained particular prominence in the past two decades. In simplified terms, its core theme is that decision makers possess a repertoire of strategies that may be more or less successful in a particular task environment (this basic idea was first put forward by Beach \& Mitchell, 1978; Payne, 1982; Payne, Bettman, \& Johnson, 1993). The particular contribution of the adaptive toolbox approach was the development of precisely described "fastand-frugal" heuristics that allow for highly accurate judgments in certain environments, despite their noteworthy simplicity (Gigerenzer, 2004). One of the simplest and yet often surprisingly accurate heuristics from the toolbox is the recognition heuristic (RH) for comparative judgments from memory.

Goldstein and Gigerenzer (2002) proposed a process model of RH use. This model presupposes that paired comparisons between objects (e.g., inferring which of two cities is more populous) are determined by whether or not objects are recognized: If exactly one of the objects is recognized - and given that recognition is associated with the criterion in the current domain - no further information (such as other probabilistic cues) is considered and the judgment is based on 
recognition alone. If, for instance, recognition is positively associated with the criterion, the recognized object is inferred to have the higher criterion value and no further cues are considered. Even though this is an example of particularly simple, ignorance-driven, one-reason decision making, it actually allows for accurate judgments in a wide variety of domains (Gigerenzer \& Goldstein, 2011; Goldstein \& Gigerenzer, 2002; Pohl, 2006).

The RH has been studied extensively and also kindled some controversy (for an overview, see Pohl, 2011), especially concerning the empirical adequacy of its one-reason decisionmaking principle (e.g., Brighton \& Gigerenzer, 2011; Hilbig \& Richter, 2011). Beyond such controversies, an emerging consensus is that the RH can account for the behavior of some individuals at times (Hilbig, 2010b; Pachur, Todd, Gigerenzer, Schooler, \& Goldstein, 2011) and that "the question is: When do people rely on the heuristic ... ?" (Gigerenzer \& Goldstein, 2011, p. 108, emphasis added). In other words, "How do people decide whether to follow the recognition heuristic or not? Although a central question for the notion that the recognition heuristic is an adaptive tool, it has received relatively little attention so far" (Pachur, 2011, p. 418).

A particularly useful starting point from which to consider this question is the adaptive decision maker framework (Payne et al. 1988; Payne et al., 1993) or cost-benefit approach (Beach \& Mitchell, 1978). The core idea of this approach is that strategy selection depends on the accuracy vis-à-vis the effort of a strategy. More specifically, the more accurate a strategy with respect to the task goals and the less effort this strategy requires, the more likely it should be selected. As a particular advantage, this framework thus allows for deriving clear-cut predictions about which conditions should foster or hamper which types of strategies. Note that, originally, the fast-and-frugal heuristics approach does not endorse the logic of an effort-accuracy trade-off in general (Gigerenzer \& Gaissmaier, 2011), actually claiming that "even if the information costs nothing, cognitive processes should still ignore a proportion of it" (Gigerenzer, 2008, p. 26). Nonetheless, several investigations of fast-and-frugal heuristics have essentially adopted the idea that accuracy and effort are vital determinants of strategy selection (Marewski \& Schooler, 2011; Newell \& Shanks, 2003; Pachur \& Hertwig, 2006; Rieskamp \& Hoffrage, 2008), providing evidence that "people select . . . simple strategies more when cognitive capacities are scarce" (Pachur et al. 2009, p. 902).

On closer inspection, several findings concerning the $\mathrm{RH}$ are indeed well-aligned with the idea of adaptive strategy selection being driven by accuracy and effort. Most importantly, a high validity of the recognition cue fosters RH use (Gigerenzer \& Goldstein, 2011; Hilbig, Erdfelder, \& Pohl, 2010; Pohl, 2006). Similarly, the larger the discrepancy in the memory strengths of recognized and unrecognized objects (which is typically strongly correlated with the recognition validity), the more likely is RH use (Castela, Kellen, Erdfelder, \& Hilbig, 2014; Erdfelder, Küpper-Tetzel, \& Mattern, 2011). Thus, the higher the accuracy that one may attain by relying on the RH, the more likely this behavior becomes. At the same time, situations rendering effort reduction more attractive or even necessary also induce choices more in line with the RH. For example, time pressure (Hilbig et al. 2012; Pachur \& Hertwig, 2006), a deliberative mode of thinking (Hilbig, Scholl, \& Pohl, 2010), and depletion of executive resources (Pohl et al. 2013) have been experimentally linked to increased reliance on the RH-in line with the general view that heuristics are particularly useful as a means of effort reduction (Shah \& Oppenheimer, 2008).

In turn, it has also been shown that data are less consistent with the RH whenever decision makers have additional knowledge at their disposal. This, too, is in line with the idea of adaptive strategy selection: The more useful one's additional knowledge and the more easily this knowledge may be integrated into a judgment, the more likely a strategy integrating multiple pieces of information is to be selected (as opposed to the RH). It is thus to be expected that, once recognized objects are conclusively known to possess a small criterion value, the $\mathrm{RH}$ is overruled by this knowledge (Oppenheimer, 2003; as explained in detail by Pachur \& Hertwig, 2006). However, even beyond conclusive criterion knowledge (which essentially renders the RH obsolete; see, e.g., Hilbig, Pohl, \& Bröder, 2009; Pachur et al. 2008), available information is typically considered: The availability of probabilistic cues beyond recognition generally reduces the degree to which choices are in line with the RH (Newell \& Fernandez, 2006; Pachur et al., 2008; Richter \& Späth, 2006). This holds even if cue values must be retrieved from memory (Bröder \& Eichler, 2006). Moreover, once cues are made available, choices, reaction times, and confidences are better accounted for by models assuming (weighted-additive) information integration rather than the RH (Glöckner \& Bröder, 2011, 2014), although these findings may hinge on the specific assumptions about reaction times and confidences for the RH. Again in line with the adaptive decision-maker framework, cue information beyond recognition is particularly sought (even at some monetary cost) whenever recognition yields only low validity (Newell \& Shanks, 2004). Finally, although there appear to be noteworthy individual differences in RH use (Pachur et al., 2008), these are also in line with the general pattern: More knowledgeable individuals tend to rely less on the RH (Hilbig \& Pohl, 2008), as do those presumably more willing to put their knowledge to a test (Hilbig, 2008).

However, despite this rather consistent picture, little is known about the role of cognitive costs in information integration and how these, in turn, influence RH use. Although consideration of further information (and thus, RH nonuse) should depend on how effortful information integration is, prior work has been mute on this issue, because investigations 
have allowed for effortless information integration. In the studies sketched above, additional cues were always reduced to and presented to participants in a simple, binary (present vs. absent) format that is particularly easy to process. Also, the sign of the correlation between cue values and the criterion was always clear a priori, with presence typically indicating a higher criterion value. Moreover, decision makers could be quite certain about the usefulness of cues, given that they were fully informed about the actual cue validity (e.g., Newell \& Fernandez, 2006, Exp. 1), had ample opportunity to learn the cue validities (e.g., Bröder \& Eichler, 2006), or were told that cue validity was high (e.g., Richter \& Späth, 2006, Exp. 3). Arguably, further cues are relatively easy to integrate and come with relatively high subjective usefulness when they are made available in such a neatly prestructured manner. Thus, it is unsurprising from an adaptive strategy selection perspective that cues would be considered to some extent (thus reducing RH use) in such situations. In turn, one might expect less consideration of further cues and more RH use whenever information integration requires increased cognitive costs.

As an example, consider comparing the US cities San Jose and Indianapolis with respect to their populations. One useful cue in this situation could be derived from the state that each of the cities is located in: Since California is the more populous state (and the one with more major cities), as compared to Indiana, one could draw the (correct) inference that San Jose is more populous. If the state information were presented alongside the city names, integrating this information might be a relatively easy feat. If, by contrast, the state information had to be retrieved from memory, this would be more difficult. More importantly, such a retrieval process would typically induce uncertainty, for example because one might be unsure whether San Jose is located in California. Finally, even if one is told (or successful at retrieving from memory) which state each of the cities is located in, there would still be uncertainty about how to construct a probabilistic cue from this information and how valid this cue might be. By contrast, one would immediately know whether and how best to use this information if one were told that - in comparing US cities with more than 500,000 inhabitants - a city located in California is more populous than a city located in any other state with a probability of about $80 \%{ }^{1}$ In this case, it is clear how to transform state information into a binary cue (California vs. other state) and how valid this cue is.

In summary, we propose that consideration of information beyond recognition (as opposed to reliance on recognition alone, and thus RH use) will become more likely, the more easily accessible this information is and the more certainty it comes with - especially concerning its predictive validity. So far, no systematic investigations have tested to what extent cue

\footnotetext{
${ }_{1}^{1}$ According to the 2010 US census; see http://en.wikipedia.org/wiki/ List_of_United_States_cities_by_population, retrieved June 2012.
}

integration versus RH use depends on the difficulty and subjective success of processing cue knowledge. However, contemporary theories of strategy selection predict such a pattern (Marewski \& Schooler, 2011), and corresponding effects have been found in work pertaining to other decision strategies: For example, Bröder and Schiffer (2003b) showed that a simpler, lexicographic strategy is more often used if cue information is more difficult to retrieve from memory (Bröder \& Schiffer, 2006b). In turn, once a cue is easily retrieved (e.g., due to high salience), it is most likely to be integrated into the judgment, even if it is not particularly valid (Platzer \& Bröder, 2012). Likewise, high processing fluency has been shown to increase the probability of cue integration (Shah \& Oppenheimer, 2007). Söllner, Bröder, and Hilbig (2013) further found that high information accessibility fosters processes of automatic information integration, whereas low accessibility (effortful information search) triggers sequential processing and, in turn, more reliance on simpler strategies. Transferring findings of this nature to the $\mathrm{RH}$, it is straightforward to hypothesize that as information integration beyond recognition becomes easier and subjectively more accurate, decision makers should tend to consider such additional information, and thus refrain from using the RH. In the following sections, we report a series of three experiments testing this prediction.

\section{Experiment 1}

\section{Method}

Material and design In this experiment, we relied on the most common paradigm for investigating the RH: comparing pairs of cities with respect to their population (Hilbig \& Pohl, 2009; Pachur et al., 2008). As materials we used large world cities, since these allowed for the consideration of a naturally available additional cue: the countries in which cities are located. Specifically, we selected a set of 20 cities on the basis of a prestudy, such that the recognition cue had a validity of about .70 , which is roughly representative of the total set of large world cities (Hilbig, 2010b). At the same time, cities were selected such that the same validity of .70 applied to the additional cue, namely whether or not a city was located in Asia (subsuming South, East, or Southeast Asia; for simplicity, referred to as the "Asia cue" in what follows). We further ensured that both cues (recognition and Asia) had approximately the same discrimination rate, rendering them equivalent not only in validity but also in terms of their a priori success rate (Newell, Rakow, Weston, \& Shanks 2004). The cities are listed in the online supplemental materials.

Importantly, we tested in an independent prestudy whether participants ( $N=40,28$ female, 12 male; 17 to 30 years of age, 
$M=22.0, S D=2.3$ ) were able to reliably judge the value of the Asia cue for each city: They were given a questionnaire containing all city names in alphabetical order and asked to indicate whether or not each city was located in South, East, or Southeast Asia. To ensure complete understanding, a footnote specified exactly which countries this region comprises. Participants provided an average proportion of $M=.75$ ( $S E$ $=.02$ ) correct judgments, which was well above chance level $[t(39)=13.7, p<.001$, Cohen's $d=2.1]$, confirming that they possessed substantial (albeit imperfect) knowledge about the country that each city is located in.

To test our hypothesis, we manipulated the ease of knowledge integration between participants in two conditions: In the control condition, which resembled the typical setup of most prior work, participants were shown pairs of city names (e.g., "Lahore-Santiago") and asked to infer which was the more populous one. No other information was provided. However, as the findings of the prestudy implied, participants could in principle construct the Asia cue in this condition - though this would require effort and come with some uncertainty. By contrast, in the knowledge condition, participants were additionally informed about the corresponding countries, which were displayed alongside the city names (e.g., "Lahore, Pakistan-Santiago, Chile"). In this condition, the Asia cue was thus made more easily accessible than in the control condition.

Across the two conditions, we predicted a decrease in $\mathrm{RH}$ use: As compared to the control condition, the knowledge condition made information more easily available, which should foster the integration of this information. Note that participants were not provided with explicit binary values of the Asia cue in the knowledge condition. As such, information integration always required at least some additional effort (viz., at a minimum, constructing the Asia cue from the country information). Also, note again that neither RH use nor information integration was the more accurate strategy a priori-since the materials ensured that both cues would be comparable in terms of validity and success.

Procedure and participants The participants completed two tasks in random order: a paired-comparison task and a recognition task. The former featured a total of 190 comparisons (from pairing the 20 cities exhaustively), displayed in random order with a 500-ms intertrial interval. In each trial, participants were asked to indicate which of the two cities was more populous by pressing one of two keys. Responses and latencies were recorded. For each correct judgment, participants gained $€ 0.04$, whereas for each false judgment they lost $€ 0.04$. However, to avoid strategy-learning effects, no feedback was provided, and participants only learned about their overall performance (and thus earnings) after the entire experiment was concluded. In the recognition task, all cities were presented one at a time, in a random order with a 500-ms intertrial interval, and participants were asked to judge whether or not they had heard of each city before the experiment. A total of 46 participants ( 37 female, nine male), between 17 and 33 years of age $(M=22.2$ years, $S D=3.9)$, were recruited at the University of Mannheim and randomly assigned to one of the conditions described above.

\section{Results and discussion}

Descriptive analyses revealed that the data were comparable to those from previous investigations of the $\mathrm{RH}$ and also across experimental conditions: Participants recognized 11.5 objects on average $(S D=2.7)$. Hence, the recognition cue had a typical mean discrimination rate of $.48(S D=.11)$, which was similar to the discrimination rate of the Asia cue (constant at .52). Moreover, the mean recognition rate did not differ between conditions $(M=.47, S D=.12$, and $M=.49, S D=.11$, for the control and the knowledge conditions, respectively) $[t(44)=0.58, p=.57$, Cohen's $d=0.17]$. The actual mean recognition validity was .68 $(S D=.10)$, and thus was similar to the validity of the Asia cue (which was constant at .68). Also, the mean recognition validity did not differ across conditions $(M=67, S D=.09$, and $M=.68, S D=.10$, for the control and knowledge conditions, respectively) $[t(42)=$ $0.51, p=.61$, Cohen's $d=0.16]$. In summary, the recognition cue was comparable to the Asia cue in terms of validity and discrimination rate, and both cues were equivalent in validity and discrimination rate across experimental conditions.

To test the main hypothesis, the data were analyzed with the $r$-model (Hilbig, Erdfelder, \& Pohl, 2010), a multinomial processing tree model (Batchelder \& Riefer, 1999; Erdfelder et al., 2009). Apart from other measures of minor importance in the present context, this model provides asymptotically unbiased estimates of the probability of RH use - that is, the probability of relying only on the recognition cue (in the sense of one-reason decision making). To achieve this, all trials are assigned to one of three cases (or trees) - namely knowledge cases (both options recognized), recognition cases (one option recognized), and guessing cases (neither option recognized). Most importantly, observable responses in recognition cases are accounted for by three latent parameters - namely the probability of using the $\mathrm{RH}$ (parameter $r$ ), the probability of valid knowledge whenever the RH is not used (parameter $b$ ), and the recognition validity (parameter $a$ ). Thus, the $r$ parameter represents the probability that judgments are based on recognition alone, without consideration of further information, as presupposed by Goldstein and Gigerenzer's (2002) RH theory. This measurement model has been validated both experimentally and through simulations (Hilbig, 2010a) and has previously been applied successfully in several studies. All analyses were performed with multiTree (Moshagen, 2010) and MPTinR (Singmann \& Kellen, 2013). 
First, as is common practice in multinomial modeling, the model was applied jointly to the data of both experimental conditions, with each condition comprising the choice frequencies aggregated across all of its participants. This full model fit the data $\left[G^{2}(2)=5.1, p=.08, \Delta \mathrm{AIC}=1.1, \Delta \mathrm{BIC}\right.$ $=-13.0]$. More importantly, maximum likelihood parameter estimates revealed that $\mathrm{RH}$ use was substantial and at a very typical level in the control condition $(\hat{r}=.69, S E=.02)$, and notably lower in the knowledge condition $(\hat{r}=.45, S E=.02)$. Confirming this descriptive pattern, a submodel restricting the two $r$ parameters to equality across conditions fit the data significantly worse $\left[\Delta G^{2}(1)=74.8, p<.001\right]$. Note that the difference between the conditions in RH use was actually larger than the effects on $r$ estimates produced by manipulations such as the instruction to use the RH (Hilbig, Erdfelder, \& Pohl, 2010).

Additionally, to ensure that the reported parameter estimates of the aggregate data mirrored the typical individual ones, we estimated the $r$-model parameters for the data of each individual participant (Hilbig, Erdfelder, \& Pohl, 2011) and tested for a difference between means. Here and in all following analyses, we used one-sided tests for all directional hypotheses. The model fit the data $\left[G^{2}(1)<3.8, p>.05\right]$ of all but seven participants, who were consequently discarded in the following individual analysis (although including them left all findings unaltered). Most importantly, the means of the individual $r$-parameter estimates closely matched the aggregate ones $(M \mathrm{~s}=.64$ and .47 for the control and knowledge conditions, respectively), and a one-sided $t$ test confirmed the influence of the between-subjects condition on the individual $r$-parameter estimates $[t(38)=1.7, p=.049$, Cohen's $d=$ 0.56]. Additionally, we computed the Bayes factor for the one-sided alternative against the null hypothesis by means of the BayesFactor R package (Rouder et al. 2009). Confirming the results above, the Bayes factor indicated evidence in favor of the alternative hypothesis of an influence of the betweensubjects condition on RH use $\left(B F_{10}=1.8\right)$, albeit only weak evidence (the data were about two times more likely under the alternative than under the null hypothesis).

Next, to investigate which specific processes or decision strategies accounted for the observed decrease in $\mathrm{RH}$ use from the control to the knowledge condition, we resorted to the outcome-based maximum likelihood classification method (Bröder \& Schiffer, 2003a), which has been successfully applied in various investigations (Bröder \& Schiffer, 2006a; Hilbig \& Moshagen, 2014; Pachur \& Olsson, 2012) including investigations of the RH very similar (in structure) to the current one (Hilbig, 2014). Specifically, we compared the likelihood of four competing decision strategies, given the choice data of each individual participant in those trials in which exactly one city was recognized. These trials can be subdivided into (i) those in which recognition and the Asia cue made the same choice predictions, (ii) those in which the Asia cue did not discriminate, and (iii) those in which the Asia cue contradicted the recognition cue in terms of choice predictions. For each of these three subcases, ${ }^{2}$ we computed how often each participant chose the recognized city.

The four competing models were the RH, an equal-weights model (EQW; i.e., "choose the option with the higher sum of cue values"), a noncompensatory Asia heuristic (ASIA; i.e., "choose the option to which the Asia cue points; if it does not discriminate, guess"), and guessing (GUESS). The RH predicts that the probabilities of choosing the recognized city should be the same across the three subcases, since the country information is claimed to be ignored altogether. EQW is an information-integration model and predicts that the probability of choosing the recognized city should be the same in subcases $\mathrm{i}$ and ii, but that guessing should occur in subcase iii, since the cue pattern favors neither of the cities. The ASIA strategy considers the Asia cue in isolation, and thus predicts equivalent choice probabilities in subcases $i$ and iii and guessing in subcase ii; note that this strategy predicts choice of the unrecognized option in subcase iii. Finally, GUESS predicts random responding in all three subcases. As is common practice (Bröder \& Schiffer, 2003a; Glöckner, 2009; Jekel et al. 2010), we determined the Bayesian information criterion (BIC; Wasserman, 2000) for each of the four strategies per individual data set and classified each participant as a user of the strategy that produced the smallest BIC. ${ }^{3}$ The raw choice frequencies of each individual and the model equations for RH, EQW, ASIA, and GUESS to be used in MPTinR are provided in the online supplemental materials.

Table 1 shows the individual-level strategy classification results per condition. As can be seen, in the control condition, a large majority of participants were best described by the $\mathrm{RH}$, whereas a negligible percentage were accounted for by the EQW strategy, and no participant was classified as an ASIA user. By contrast, in the knowledge condition, the percentage of participants best classified by the RH dropped substantially, accompanied by a considerable increase in the percentage of participants best accounted by the EQW strategy. Again, a few participants seemed to be guessing, and no participant was classified as an ASIA user. The difference in percentages of RH versus EQW users between conditions was significant, as indicated by a $\chi^{2}$ test of independence $\left[\chi^{2}(1)=4.8, p=.03\right]$. These findings confirm the $r$-model analyses and further specify the results: $\mathrm{RH}$ nonuse could be accounted for by a fully

\footnotetext{
${ }^{2}$ Note again that, strictly speaking, the Asia cue was not provided explicitly in any of the conditions. However, it could be inferred in both conditions (including the control condition - as confirmed by the prestudy), though with varying effort and certainty - as is the logic of the present experiment.

${ }^{3}$ In line with prior recommendations (Bröder \& Schiffer, 2003a; Glöckner, 2009), models/strategies were discarded for classification if the average proportion of strategy-inconsistent choices was larger than .30. However, the pattern of results was independent of this constraint.
} 
Table 1 Percentages of participants classified as users of the four competing strategies, separately for each experimental condition

Experiment 1 / Experiment 2

\begin{tabular}{lccc}
\hline & Control & Knowledge & Certainty \\
RH & $77 \% / 85 \%$ & $50 \% / 42 \%$ & $-/ 10 \%$ \\
EQW & $5 \% / 5 \%$ & $27 \% / 42 \%$ & $-/ 65 \%$ \\
ASIA & $0 \% / 5 \%$ & $0 \% / 5 \%$ & $-/ 10 \%$ \\
GUESS & $18 \% / 5 \%$ & $23 \% / 11 \%$ & $-/ 15 \%$ \\
\hline
\end{tabular}

Strategy classifications were highly reliable, since the percentage of classifications that yielded strong or very strong evidence, in terms of the Bayesian posterior probability of the classified model relative to the competing ones (Wagenmakers, 2007), was $75 \%$. Also, disregarding models that misfit the data $(p<.05)$ in absolute terms - to rule out falsely classifying data that were most likely produced by a strategy outside the consideration set (Moshagen \& Hilbig, 2011) —did not alter the main pattern.

RH Recognition Heuristic, EQW Equal Weights Strategy, ASIA Asia Heuristic, GUESS guessing (choice at random)

specified information integration model - namely, one that bases choices on the sum of (unweighted) cue values.

Finally, to test whether convergent evidence could also be obtained via process measures, we analyzed participants' decision times, separately for participants classified as RH users and those classified as EQW users (for a similar approach, see Bröder \& Gaissmaier, 2007). Specifically, we contrasted trials in which the Asia cue pointed to the same choice option as recognition against those in which the Asia cue contradicted recognition. The RH theory predicts that decision times should be independent of whether or not the Asia cue confirms or contradicts recognition, because this additional information is ignored (Hilbig \& Pohl, 2009). EQW, by contrast, predicts that cases of conflict should require more time, since an additional step must be taken (viz. guessing) whenever a choice cannot be made on the basis of the sum of cue values per choice option. For each participant, we analyzed decision times, ${ }^{4}$ conditional upon the type of trial. Confirming the result above, the decision times of participants classified as RH users did not differ depending on whether or not the Asia cue was aligned with recognition $(M=1,341 \mathrm{~ms}, S E=115$, and $M=1,275 \mathrm{~ms}$, $S E=59$, respectively) $[t(27)=0.07, p=.47$, Cohen's $d=0.01$, $\left.B F_{10}=0.21\right]$. By contrast, individuals classified as EQW users showed longer decision times when cues conflicted than when they were aligned $(M=2,244 \mathrm{~ms}, S E=267$, and $M=1,809$ $\mathrm{ms}, S E=183$, respectively) $[t(6)=2.74, p=.02$, Cohen's $d=$ $\left.1.0, B F_{10}=5.2\right]$. In sum, decision times were in line with the process predictions of the strategies, thereby lending further support to the main conclusions.

Overall, the findings of Experiment 1 confirm the basic hypothesis that easily available additional knowledge will

\footnotetext{
${ }^{4}$ To account for nonnormality, the tests were based on mean logtransformed decision times. For ease of interpretation, descriptives are given as median decision times.
}

indeed be considered, thus reducing RH use. In principle, information beyond recognition was available in both conditions (as was shown by the prestudy, participants could have generated the country information in the control condition). However, making it easily accessible - that is, relieving participants of the burden of retrieving it - substantially increased information integration. Plausibly, maximum likelihood strategy classification - which, in turn, was confirmed by reaction time analyses - implied that the reduction in RH use can be accounted for by a simple alternative model: an equal-weights strategy. The influence of additional information was uncommonly strong, although participants were not provided with binary cue values - unlike in previous experiments (Goldstein \& Gigerenzer, 2002; Newell \& Fernandez, 2006; Richter \& Späth, 2006). Note that the difference in single-cue reliance on recognition between the control condition and the one comprising immediate accessibility of country knowledge was larger than the effects of time pressure (Hilbig et al., 2012) or cognitive resource depletion (Pohl et al., 2013) found in previous studies.

However, one may argue that the effect demonstrated in Experiment 1 was merely a minor extension of such previous experiments, showing that information provided will be used even if it is not in a binary format and the cue direction is not clear a priori. Even so, we did not directly manipulate the degree to which information integration beyond recognition was easier and subjectively more accurate. As such, it remains an open question whether the probability of RH use actually depends on the effort and subjective certainty associated with information integration. To test this claim more conclusively, we devised two further experiments. The logic of both was to alter the basic effect demonstrated above. Specifically, in Experiment 2, we sought to further augment the effect by reducing the uncertainty associated with information integration. In turn, Experiment 3 was designed to reduce or eliminate the effect by rendering information integration more cognitively demanding.

\section{Experiment 2}

Method

Material and design To maximize comparability, the second experiment was an exact replication of Experiment 1 in terms of the material and basic setup. That is, we again implemented the same control and knowledge conditions, identical to those reported above. As before, in the control condition, participants worked on the recognition and judgment tasks without further information. Thus, the Asia cue was in principle constructible, but this would require cognitive effort and come with some uncertainty. Again, in the knowledge condition, use of the Asia cue was made less effortful than in the control 
condition by directly displaying the country information. More importantly, we extended Experiment 1 by adding a third experimental condition. In this certainty condition, country information was again provided (exactly as in the knowledge condition). Additionally, however, participants were informed before the comparison task that cities located in Asian countries (subsuming South, East, and Southeast Asia) had a $70 \%$ chance of being more populous than cities not located in this region. Consequently, any uncertainty as to how best to integrate the country information (and how useful this would be) was eliminated as compared to the knowledge condition.

Across the three conditions, we predicted a decrease in $\mathrm{RH}$ use: In comparing the control condition to the knowledge condition, we expected to replicate the decrease in RH use found in Experiment 1. In turn, as compared to the knowledge condition, participants in the certainty condition additionally knew how best to restructure the information given and how useful (in terms of validity) the resulting cue would be. Thus, we hypothesized a further decrease in RH use.

Procedure and participants Exactly as in Experiment 1, participants completed a paired-comparison task and a recognition task (both with a 1,000-ms intertrial interval) in random orderusing the same cities (and country names) as in Experiment 1. For each correct judgment in the paired-comparison task, $€ 0.05$ was added to their earnings, whereas each false judgment lost them $€ 0.05$. A total of 61 participants ( 41 female, 20 male), 19 to 30 years of age $(M=22.1$ years, $S D=2.6)$, were randomly assigned to the three conditions outlined above.

\section{Results and discussion}

As before, the data were comparable to those from previous investigations of the $\mathrm{RH}$, and also across the experimental conditions: Participants recognized 12.4 objects on average $(S D=2.4)$, implying a typical mean discrimination rate of .46 $(S D=.11)$, which was comparable to the discrimination rate of the Asia cue (.52) and did not differ across the three experimental conditions $(M=.46, S D=.12 ; M=.43, S D=.12$; and $M=.48, S D=.06$, for the control, knowledge, and certainty conditions, respectively) $\left[F(2,58)=1.5, p=.24, \eta_{\mathrm{p}}{ }^{2}=.05\right]$. The actual mean recognition validity $(M=.68, S D=.11)$ was equivalent to the validity of the Asia cue (which was constant at .68) and did not differ significantly across conditions $(M=$ $.69, S D=.11 ; M=.70, S D=.11$; and $M=.65, S D=.10$, for the control, knowledge, and certainty conditions, respectively) $\left[F(2,57)=1.3, p=.29, \eta_{\mathrm{p}}{ }^{2}=.04\right]$. In summary, the recognition cue yielded findings comparable to those from typical investigations, was approximately equivalent to the Asia cue in terms of validity and discrimination rate, and also comparable across experimental conditions.
To test the main hypothesis, the $r$-model was applied jointly to the data of all three experimental conditions, with each condition comprising the choice frequencies aggregated across all its participants. This full model fit the data $\left[G^{2}(3)=\right.$ $7.1, p=.07, \Delta \mathrm{AIC}=1.1, \Delta \mathrm{BIC}=-21.0]$. Parameter estimates revealed an almost perfect replication of Experiment 1, in that RH use was again substantial and at a typical level in the control condition $(\hat{r}=.70, S E=.02)$, and intermediate in the knowledge condition $(\hat{r}=.50, S E=.02)$. More importantly, $\mathrm{RH}$ use was further decreased and uncommonly low in the certainty condition $(\hat{r}=.30, S E=.02)$. A submodel constraining the three $r$ parameters to equality across conditions yielded severe misfit $\left[\Delta G^{2}(2)=180.2, p<.001\right]$, thus confirming the difference between the $r$-parameter estimates statistically. Moreover, every pair-wise difference between conditions was also significant [all $\Delta G^{2} \mathrm{~s}(1)>35.1$, all $p \mathrm{~s}<$ $.001]$. Once more, each of these pair-wise differences was considerably larger than the effects typically found in prior experiments.

Again, to ensure that the aggregate parameter estimates mirrored the typical individual ones, the $r$-model was applied to the data of each individual participant. The model fit was not satisfactory $\left[G^{2}(1)>3.8, p<.05\right]$ for four participants, who were consequently discarded in the following individual analyses (though, once again, including them left all findings unaltered). Confirming the aggregate analysis, the means of the individual $r$-parameter estimates closely matched the aggregate ones $(M s=.68, .45$, and .33 , for the control, knowledge, and certainty conditions, respectively), and a one-way analysis of variance confirmed the influence of the betweensubjects condition on the individual $r$-parameter estimates $\left[F(2,54)=8.2, p=.001, \eta_{\mathrm{p}}^{2}=.23\right]$. Follow-up $t$ tests (and the corresponding Bayes factors; cf. Rouder et al., 2009) showed that use of the RH was substantially higher in the control than in the knowledge condition $[t(37)=2.5, p=.01$, Cohen's $d=0.81, B F_{10}=6.3$, and was decisively higher in the control than in the certainty condition $[t(37)=4.2, p<$ .001 , Cohen's $\left.d=1.4, B F_{10}=299.9\right]$. However, despite a small-to-medium-sized effect (Cohen's $d=0.43$ ), the decrease in RH use between the knowledge and certainty conditions did not reach a conventional level of significance $[t(34)=1.3, p=$ .11]. Similarly, the Bayes factor (of 1.03) indicated that neither hypothesis was superior. In summary, the finding that RH use decreased across the three conditions was mostly supported by the individual-level analysis.

As in Experiment 1, we again tested whether specific decision strategies could account for the observed pattern in RH use. Repeating the outcome-based maximum likelihood strategy classification method (exactly as described above; again, the raw data are available in the online supplemental materials) indeed confirmed this expectation and the findings of Experiment 1. As can be seen in Table 1, a large majority in the control condition were best described by the RH. By 
contrast, in the knowledge condition, individuals classified as users of the RH versus a knowledge integration strategy (EQW) were about equally prevalent. Finally, in the certainty condition, practically no participant was most likely to have used the RH, whereas a majority appeared to be integrating cues. The difference in the percentages of RH versus EQW users between conditions was significant $\left[\chi^{2}(2)=22.9, p<\right.$ $.001]$. Overall, the findings again confirmed the $r$-model analyses and further specified the results: RH nonuse was due to information integration that corresponded to a strategy assigning equal weights to recognition and country information. At the same time, we found no indication of participants resorting to a strategy that placed more importance on the country information than on recognition (as would have been implied by the alternative, noncompensatory ASIA strategy).

Decision times were again analyzed to test whether these converged with the strategy classification results. Confirming the result above, the decision times of participants classified as RH users did not differ depending on whether or not the Asia cue was aligned with recognition $(M=1,679 \mathrm{~ms}, S E=125$, and $M=1,783 \mathrm{~ms}, S E=172$, respectively) $[t(26)=0.73, p=$ .23 , Cohen's $d=0.14, B F_{10}=0.39$ ]. By contrast, individuals classified as EQW users showed shorter decision times when cues were aligned than when they conflicted $(M=1,994 \mathrm{~ms}$, $S E=201$, and $M=2,342 \mathrm{~ms}, S E=198$, respectively) $[t(21)=$ 2.71, $p<.01$, Cohen's $\left.d=0.58, B F_{10}=7.8\right]$.

Overall, we compared RH use across three experimental conditions that differed in (a) the accessibility of information beyond recognition (replicating Exp. 1) and, in addition, (b) the certainty associated with the predictive usefulness of this information. The latter extends Experiment 1 with the hypothesis that the effect of available information should be boosted and thus RH use further decreased. Descriptive results were in line with these predictions both on the aggregate and the individual level. Indeed, when information beyond recognition was both easily accessible and known to be useful, RH use occurred with uncommonly low prevalence that was comparable to situations in which recognition has no predictive validity whatsoever [Hilbig, Erdfelder, \& Pohl, 2010, Exp. 7(b)]. Again, decreases in RH use were accompanied by increases in the degree to which a fully specified information-integration model - an equal-weights strategyaccounted for the data. As such, participants were not merely guessing or shifting to a different non-compensatory heuristic; rather, they most likely integrated the available pieces of information. In summary, the present results support the idea of adaptive decision making: The less effort is required to integrate additional information and the more subjectively dependable this information is, the fewer judgments are based on recognition alone.

However, it could be criticized that Experiment 2 does not provide conclusive insight on the role of cognitive costs. Although it is indubitable that cognitive costs decreased across the three conditions, one could alternatively argue that providing additional information (as in the knowledge condition) and pointing out how best to use this information (as in the certainty condition) produces demand effects. Thus, to test more conclusively whether the cognitive costs associated with information integration are the crucial factor, it seemed necessary to manipulate these costs more directly while holding the information itself constant. To this end, we conducted a third experiment. Specifically, the main goal of the third experiment was to test whether the basic effect (of knowledge availability) observed in Experiments 1 and 2 can also be reduced or even eliminated (rather than boosted as in Exp. 2) once information integration is cognitively costly.

\section{Experiment 3}

Method

Material and design In this experiment, we manipulated the cognitive effort of knowledge integration between participants in three conditions. Again, there were a control and a knowledge condition - similar to those in the previous two experiments. As before, participants in the knowledge condition were informed about the country that each city belonged to, facilitating knowledge integration as compared to the control condition (in which, once again, no additional information was provided). In addition, we implemented an effort condition, in which participants were again informed about the corresponding country. However, unlike in the knowledge condition, the country names were scrambled (random order of letters) and Qs and Ws (letters that did not appear in any of the country names) were added at random positions to the letter strings. The numbers of Qs and Ws added were such that all transformed country names became equivalent in length, making it impossible to infer the country from the length of the letter sequence. For example, a comparison was "Lahore-Santiago" in the control condition, "Lahore, Pakistan-Santiago, Chile" in the knowledge condition, and "Lahore, WPAQSWNAQTWKWWIWQ-Santiago, CWWWEQHQQIQWWQWLQ" in the effort condition. Participants were told that the letter sequences contained the country names, though scrambled and with Qs and Ws added. Consequently, in the effort condition, the country information was no less available than in the knowledge condition, but it was clearly less easily accessible. ${ }^{5}$ In other words, the difficulty of extracting the information from the letter sequences

\footnotetext{
$\overline{5}$ In a small prestudy, we asked participants to unscramble such sequences and identify the country name. On the basis of the responses, we retained only country names (and thus sequences) for the experiment that could be solved by a majority of participants. The median time to solve a sequence was $M D=6.5 \mathrm{~s}$.
} 
on the screen made information integration effortful as compared to the knowledge condition.

Expecting to replicate the findings of Experiments 1 and 2, we predicted a decrease in RH use from the control to the knowledge condition. More importantly, this basic effect should be reduced or even eliminated in the effort condition: As compared to the knowledge condition, the effort condition increased the effort of knowledge integration. Although the same information was available, it came at an increased (cognitive) cost. Thus, RH use should increase from the knowledge to the effort condition - possibly up to the level found in the control condition if participants decided to ignore the additional information altogether.

Contrary to the experiments above, we did not resort to the typical exhaustive pairing of a relatively small set of options (that consequently reappeared many times in the paired comparisons) - because this might motivate participants to solve the difficult scrambled letter sequences and reuse the solution in many subsequent comparisons. Instead, 84 pairs of cities were randomly created from the same domain as in Experiments 1 and 2 (large world cities), ensuring that each city appeared only twice in total. We selected only cities for which a brief prestudy indicated that people could, in principle, solve the permutations and extract the country name from the letter sequence. Also, the selection ensured that the validity of the recognition and Asia cues was again approximately .70 and that both had approximately the same discrimination rate. The full list of paired comparisons used can be found in the online supplemental materials.

Procedure and participants The paired-comparison and recognition tasks were exactly the same as in the previous experiments, except for the difference in materials. For each correct judgment in the comparison task, participants received $€ 0.08$, whereas they lost $€ 0.08$ for each false judgment. A total of 96 participants (59 female, 37 male), between 18 and 37 years of age $(M=22.8$ years, $S D=4.2)$, were recruited at the University of Mannheim and randomly assigned to one of the three conditions outlined above.

\section{Results and discussion}

In line with our goals in selecting the material, the recognition and Asia cues again were comparable in terms of validity and discrimination rate. Also, no notable differences emerged between the experimental conditions: Participants recognized 50.7 objects out of 84 , on average $(S D=7.5)$. The recognition cue had a mean discrimination rate of $.38(S D=.05)$, comparable to the discrimination rate of the Asia cue (.36), and did not differ significantly between conditions $(M=.38, S D=.05$;
$M=.38, S D=.05$; and $M=.39, S D=.05$ for the control, knowledge, and effort conditions, respectively) $[F(2,93)=$ $\left.0.60, p=.55, \eta_{\mathrm{p}}{ }^{2}=.01\right]$. Likewise, the mean recognition validity was $.68(S D=.06)$, and thus was comparable to the validity of the Asia cue (.70). Also, the mean recognition validity did not differ significantly across conditions $(M=$ $.70, S D=.05 ; M=.67, S D=.06$; and $M=.68, S D=.07$, for the control, knowledge, and effort conditions, respectively) $\left[F(2,93)=2.8, p=.06, \eta_{\mathrm{p}}{ }^{2}=.06\right]$.

To test the hypotheses, we again applied the $r$-model jointly to the data of all three experimental conditions. This full model fit the data $\left[G^{2}(3)=3.3, p=.34, \Delta \mathrm{AIC}=-2.7, \Delta \mathrm{BIC}\right.$ $=-23.7]$. Maximum likelihood estimates supported the hypotheses: First, as in the previous experiments, RH use was substantial in the control condition $(\hat{r}=.58, S E=.03)$ and substantially lower in the knowledge condition $(\hat{r}=.35, S E=$ $.03)$. The difference between the two conditions was significant, since a submodel restricting the two $r$ parameters to equality yielded severe misfit $\left[\Delta G^{2}(1)=30.2, p<.001\right]$. Thus, although these estimates of RH use were lower than those found in Experiments 1 and 2 (which may have been due to the different materials), the basic effect replicated with a similar magnitude. More importantly, RH use was substantial in the effort condition $(\hat{r}=.54, S E=.03)$. In line with the hypothesis, it differed significantly from the level of RH use in the knowledge condition $\left[\Delta G^{2}(1)=22.0, p<.001\right]$, whereas it did not differ from the level of RH use in the control condition $\left[\Delta G^{2}(1)=0.80, p=.37\right]$.

Once again, we compared these aggregate estimates to the corresponding means of the individual data (estimating the $r$ model parameters for the data of each individual participant). The model fit the data $\left[G^{2}(1)<3.8, p>.05\right]$ of all but seven participants, who were consequently discarded in the following individual analysis (again, including these individuals left the findings unaltered). The individual analysis confirmed the findings reported above $(M \mathrm{~s}=.59, .40$, and .55 , for the control, knowledge, and effort conditions, respectively), and a one-way analysis of variance confirmed the betweensubjects condition effect on the individual $r$-parameter estimates $\left[F(2,86)=4.6, p=.01, \eta_{\mathrm{p}}^{2}=.10\right]$. Follow-up $t$ tests (and the corresponding Bayes factors) further supported the results: RH use was substantially higher in the control than in the knowledge condition $[t(58)=2.9, p<.01$, Cohen's $d=$ $0.77, B F_{10}=16.4$ ], thus showing the basic effect. As before, RH use was also considerably higher in the effort than in the knowledge condition $[t(57)=2.2, p=.02$, Cohen's $d=0.58$, $\left.B F_{10}=3.7\right]$. Since we had no clear predictions concerning the direction of the effect when comparing the control and effort conditions, we used a two-sided test in this case. As in the aggregate analysis, RH use did not differ between the control and effort conditions $[t(57)=0.63, p=.53$, Cohen's $d=0.17$, $\left.B F_{10}=0.31\right]$. This Bayes factor of 0.31 implied positive evidence in favor of the null hypothesis that there was no 
difference between conditions; that is, the data were more than three times as likely under the null hypothesis than under the (nondirectional) alternative.

Note that the maximum-likelihood strategy classification was not feasible for Experiment 3, because the mode of selecting items - to ensure that objects were not repeated more than once across trials - resulted in a strong imbalance between subcases i, ii, and iii (see above) and, more problematically still, too few instances of subcase $\mathrm{i}$ for most individuals. However, little might have been gained by this analysis in any case, because its primary advantage was to account for nonuse of the RH (in Exps. 1 and 2), whereas the present experiment was set up to demonstrate a return to the original level of $\mathrm{RH}$ use under cognitive effort. In this situation, the strategy classification cannot provide insight beyond the $r$-model analysis - apart from merely replicating the result from Experiments 1 and 2 that the decrease in RH use from the control to the knowledge condition was due to information integration.

Overall, the findings of Experiment 3 thus (i) replicate the difference between the control condition and the knowledge condition in Experiments 1 and 2 and, more importantly, (ii) confirm that this effect is essentially eliminated, once information integration requires cognitive effort. Importantly, the information itself was equally available in the knowledge and effort conditions, and participants were fully aware of this. Thus, the difference between the two conditions in RH use could be attributed to participants refraining from actually exerting the effort required to make use of the information in the effort condition. In fact, in the effort condition, RH use was practically equivalent to the level observed in the control condition, and thus a situation in which participants would have been forced to retrieve or generate the additional information from memory.

\section{General discussion}

Countless everyday judgments and decisions of both laypeople and professionals are formed under circumstances of fundamental uncertainty. In an attempt to further our understanding of the vital question of which cognitive processes underlie such judgments and decisions (Weber \& Johnson, 2009), frameworks assuming that people possess a repertoire of available strategies have been put forward (Payne et al., 1993). Prominent among these is the fast-and-frugal heuristics approach (Gigerenzer, 2004), which specifies several simple and yet often accurate process models for judgments and decisions (for criticism, however, see Dougherty et al. 2008).

One heavily studied strategy derived from the fast-andfrugal heuristics approach is the recognition heuristic $(\mathrm{RH}$; Goldstein \& Gigerenzer, 2002), which presupposes that probabilistic inferences are based on recognition of options in isolation, that is, without consideration of further probabilistic cues (for recent overviews, see Gigerenzer \& Goldstein, 2011; Hilbig, 2010b; Pachur et al., 2011; Pohl, 2011). Despite some remaining controversy on how pervasive such ignorance-based, one-reason decision-making is (Brighton \& Gigerenzer, 2011; Hilbig \& Richter, 2011), an increasing number of studies have investigated the conditions fostering or hampering reliance on the RH. So far, the available evidence is mostly in line with predictions derived from the notion of adaptive strategy selection (Payne et al., 1988, 1993); that is, the higher the accuracy of the RH and the stronger the need for effort reduction, the more closely the observable behavior corresponds to the predictions of the RH (e.g., Hilbig, Erdfelder, \& Pohl, 2010; Hilbig et al., 2012; Pachur \& Hertwig, 2006; Pohl, 2006; Pohl et al., 2013). In turn, the availability of knowledge beyond recognition appears to foster information integration, and thus nonuse of the RH (e.g., Bröder \& Eichler, 2006; Glöckner \& Bröder, 2011; Newell \& Fernandez, 2006; Pachur et al., 2008; Richter \& Späth, 2006). However, no investigations have critically assessed whether and how the ease and certainty of knowledge integration determine strategy selection.

In a series of three experiments, we thus tested the prediction that information integration (as opposed to ignorancebased, one-cue decision making) is fostered as information becomes more easily accessible and subjectively more accurate. In other words, we tested whether the cognitive effort associated with information integration impacts $\mathrm{RH}$ use. Across the experiments we consistently found that providing additional information - though this was not in binary format and came without a clear cue-criterion relation, unlike in prior experiments (e.g., Bröder \& Eichler, 2006; Newell \& Fernandez, 2006; Richter \& Späth, 2006) —substantially reduced single-cue reliance on recognition and thus RH use. Indeed, there was noteworthy consistency in the observed effects (i.e., changes in the $r$-parameter estimates) across the three experiments: The differences in RH use between the control and the knowledge conditions were $.23, .20$, and .23 across Experiments 1-3, respectively.

Moreover, two experiments demonstrated that this basic effect could be altered in a way predicted by the notion that accessibility and subjective accuracy of information determine the probability of this information being integrated: In Experiment 2, RH use was further reduced once participants were additionally given a hint on how best to restructure the additional information and how accurate this would be. In Experiment 3, the knowledge effect was essentially eliminated (and thus RH use restored to the same level as observed in the control condition) once information was less accessible and had to be effortfully distilled from scrambled letter sequences.

Together, the results on $\mathrm{RH}$ use are well in line with the predictions derived from an adaptive strategy selection framework (Marewski \& Schooler, 2011; Payne et al., 1993) and with the idea of an effort-accuracy trade-off: Cue integration 
(as opposed to ignorance-based, one-cue decision making) became more likely, the less effort it required. At the same time, the present setup slightly favored cue integration in terms of accuracy. By definition, in a compensatory environment, a compensatory strategy (such as an equal-weights rule that simply counts the sum of cue values for each choice option) will at least marginally outperform a noncompensatory rule such as the RH (Hogarth \& Karelaia, 2007). In the present experiments, the environment was indeed compensatory (since the recognition and Asia cues were equivalent in validity), implying that cue integration was indeed the superior strategy in terms of accuracy. Thus, it is fully in line with the notion of an adaptive effort-accuracy trade-off that we observed a shift toward cue integration whenever this was associated with decreasing effort and increasing certainty, as well as a shift back to single-cue reliance on recognition whenever information integration imposed cognitive costs.

As a limitation of this study, it should be noted that the additional information that our participants could integrate was not, strictly speaking, information that had to be retrieved from memory (which some have considered an important precondition for the RH; Pachur et al., 2008). Nonetheless, the to-be-integrated information was also not explicitly provided in a neatly prestructured fashion: Even in the condition that showed lowest $\mathrm{RH}$ use, participants were still forced to restructure the information provided. Thus, information integration always required some additional effort beyond reliance on recognition alone. As such, it is unlikely that the task rendered information integration more attractive a priori. Also, the information came in a natural form compatible with real-life situations (e.g., US cities are usually named together with the state that they are located in-which holds even for unrecognized city names). Finally, it should also be noted that our materials were deliberately selected to ensure that recognition and the additional information yielded comparable predictive validities and success rates. The recognition validity was also representative of the full domain from which the objects were sampled. The validity of the Asia cue in the cities used herein was somewhat larger than this cue's validity in the full domain from which the objects were sampled. However, if anything, this would have favored the RH (since the recognition cue was the more valid one in the full domain); in turn, the finding of equal-weights information integration can be considered a particularly strong one. At the very least, the Asia cue was not favored a priori.

In summary, the present work further specifies the determinants of fast-and-frugal decision making based on recognition. In line with the notion of adaptive strategy selection, the reported experiments show that information integration becomes more prevalent as information is more easily accessible and comes with higher subjective certainty.
Author note The research reported in this article was supported by grants from the German Research Foundation (DFG; Grant Nos. ER 224/ 2-1 and ER 224/2-2).

\section{References}

Batchelder, W. H., \& Riefer, D. M. (1999). Theoretical and empirical review of multinomial process tree modeling. Psychonomic Bulletin \& Review, 6, 57-86. doi:10.3758/BF03210812

Beach, L. R., \& Mitchell, T. R. (1978). A contingency model for the selection of decision strategies. Academy of Management Review, 3, 439-449.

Brighton, H., \& Gigerenzer, G. (2011). Towards competitive instead of biased testing of heuristics: A reply to Hilbig and Richter (2011). Topics in Cognitive Science, 3, 197-205. doi:10.1111/j.1756-8765. 2010.01124.x

Bröder, A., \& Eichler, A. (2006). The use of recognition information and additional cues in inferences from memory. Acta Psychologica, 121, 275-284.

Bröder, A., \& Gaissmaier, W. (2007). Sequential processing of cues in memory-based multiattribute decisions. Psychonomic Bulletin \& Review, 14, 895-900.

Bröder, A., \& Schiffer, S. (2003a). Bayesian strategy assessment in multiattribute decision making. Journal of Behavioral Decision Making, 16, 193-213.

Bröder, A., \& Schiffer, S. (2003b). "Take the best" versus simultaneous feature matching: Probabilistic inferences from memory and effects of representation format. Journal of Experimental Psychology. General, 132, 277-293. doi:10.1037/0096-3445.132.2.277

Bröder, A., \& Schiffer, S. (2006a). Adaptive flexibility and maladaptive routines in selecting fast and frugal decision strategies. Journal of Experimental Psychology. Learning, Memory, and Cognition, 32, 904-918.

Bröder, A., \& Schiffer, S. (2006b). Stimulus format and working memory in fast and frugal strategy selection. Journal of Behavioral Decision Making, 19, 361-380.

Brunswik, E. (1955). Representative design and probabilistic theory in a functional psychology. Psychological Review, 62, 193-217. doi:10. 1037/h0047470

Castela, M., Kellen, D., Erdfelder, E., \& Hilbig, B. E. (2014). The impact of subjective recognition experiences on recognition heuristic use: A multinomial processing tree approach. Psychonomic Bulletin \& Review, 21, 1131-1138. doi:10.3758/s13423-014-0587-4

Dougherty, M. R., Franco-Watkins, A. M., \& Thomas, R. (2008). Psychological plausibility of the theory of probabilistic mental models and the fast and frugal heuristics. Psychological Review, 115, 199-213. doi:10.1037/0033-295X.115.1.199

Erdfelder, E., Auer, T.-S., Hilbig, B. E., Aßfalg, A., Moshagen, M., \& Nadarevic, L. (2009). Multinomial processing tree models: A review of the literature. Zeitschrift für Psycologie. Journal of Psychology, 217, 108-124. doi:10.1027/0044-3409.217.3.108

Erdfelder, E., Küpper-Tetzel, C. E., \& Mattern, S. D. (2011). Threshold models of recognition and the recognition heuristic. Judgment and Decision Making, 6, 7-22.

Gigerenzer, G. (2001). The adaptive toolbox. In G. Gigerenzer \& R. Selten (Eds.), Bounded rationality: The adaptive toolbox (pp. 3750). Cambridge, MA: MIT Press.

Gigerenzer, G. (2004). Fast and frugal heuristics: The tools of bounded rationality. In D. J. Koehler \& N. Harvey (Eds.), Blackwell handbook of judgment and decision making (pp. 62-88). Malden, MA: Blackwell.

Gigerenzer, G. (2008). Why heuristics work. Perspectives on Psychological Science, 3, 20-29. 
Gigerenzer, G., \& Gaissmaier, W. (2011). Heuristic decision making. Annual Review of Psychology, 62, 451-482. doi:10.1146/annurevpsych-120709-145346

Gigerenzer, G., \& Goldstein, D. G. (2011). The recognition heuristic: A decade of research. Judgment and Decision Making, 6, 100-121.

Gigerenzer, G., Hoffrage, U., \& Kleinbölting, H. (1991). Probabilistic mental models: A Brunswikian theory of confidence. Psychological Review, 98, 506-528. doi:10.1037/0033-295X.98.4.506

Gigerenzer, G., \& Selten, R. (2001). Bounded rationality: The adaptive toolbox. Cambridge, MA: MIT Press.

Glöckner, A. (2009). Investigating intuitive and deliberate processes statistically: The multiple-measure maximum likelihood strategy classification method. Judgment and Decision Making, 4, 186-199.

Glöckner, A., \& Bröder, A. (2011). Processing of recognition information and additional cues: A model-based analysis of choice, confidence, and response time. Judgment and Decision Making, 6, 23-42.

Glöckner, A., \& Bröder, A. (2014). Cognitive integration of recognition information and additional cues in memory-based decisions. Judgment and Decision Making, 9, 35-50.

Goldstein, D. G., \& Gigerenzer, G. (2002). Models of ecological rationality: The recognition heuristic. Psychological Review, 109, 75-90. doi:10.1037/0033-295X.109.1.75

Hilbig, B. E. (2008). Individual differences in fast-and-frugal decision making: Neuroticism and the recognition heuristic. Journal of Research in Personality, 42, 1641-1645.

Hilbig, B. E. (2010a). Precise models deserve precise measures: A methodological dissection. Judgment and Decision Making, 5, 272-284.

Hilbig, B. E. (2010b). Reconsidering "evidence" for fast-and-frugal heuristics. Psychonomic Bulletin \& Review, 17, 923-930. doi:10.3758/ PBR.17.6.923

Hilbig, B. E. (2014). On the role of recognition in consumer choice: A model comparison. Judgment and Decision Making, 9, 51-57.

Hilbig, B. E., Erdfelder, E., \& Pohl, R. F. (2010). One-reason decision making unveiled: A measurement model of the recognition heuristic. Journal of Experimental Psychology. Learning, Memory, and Cognition, 36, 123-134. doi:10.1037/a0017518

Hilbig, B. E., Erdfelder, E., \& Pohl, R. F. (2011). Fluent, fast, and frugal? A formal model evaluation of the interplay between memory, fluency, and comparative judgments. Journal of Experimental Psychology. Learning, Memory, and Cognition, 37, 827-839. doi: 10.1037/a0022638

Hilbig, B. E., Erdfelder, E., \& Pohl, R. F. (2012). A matter of time: Antecedents of one-reason decision making based on recognition. Acta Psychologica, 141, 9-16. doi:10.1016/j.actpsy.2012.05.006

Hilbig, B. E., \& Moshagen, M. (2014). Generalized outcome-based strategy classification: Comparing deterministic and probabilistic choice models. Psychonomic Bulletin \& Review, 21, 1431-1443.

Hilbig, B. E., \& Pohl, R. F. (2008). Recognizing users of the recognition heuristic. Experimental Psychology, 55, 394-401.

Hilbig, B. E., \& Pohl, R. F. (2009). Ignorance- versus evidence-based decision making: A decision time analysis of the recognition heuristic. Journal of Experimental Psychology. Learning, Memory, and Cognition, 35, 1296-1305.

Hilbig, B. E., Pohl, R. F., \& Bröder, A. (2009). Criterion knowledge: A moderator of using the recognition heuristic? Journal of Behavioral Decision Making, 22, 510-522.

Hilbig, B. E., \& Richter, T. (2011). Homo heuristicus outnumbered: Comment on Gigerenzer and Brighton (2009). Topics in Cognitive Science, 3, 187-196. doi:10.1111/j.1756-8765.2010.01123.x

Hilbig, B. E., Scholl, S. G., \& Pohl, R. F. (2010). Think or blink-Is the recognition heuristic an "intuitive" strategy? Judgment and Decision Making, 5, 300-309.

Hogarth, R. M., \& Karelaia, N. (2007). Heuristic and linear models of judgment: Matching rules and environments. Psychological Review, $114,733-758$.
Jekel, M., Nicklisch, A., \& Glöckner, A. (2010). Implementation of the multiple-measure maximum likelihood strategy classification method in R: addendum to Glöckner (2009) and practical guide for application. Judgment and Decision Making, 5, 54-63.

Marewski, J. N., \& Schooler, L. J. (2011). Cognitive niches: An ecological model of strategy selection. Psychological Review, 118, 393437.

Moshagen, M. (2010). multiTree: A computer program for the analysis of multinomial processing tree models. Behavior Research Methods, 42, 42-54. doi:10.3758/BRM.42.1.42

Moshagen, M., \& Hilbig, B. E. (2011). Methodological notes on model comparisons and strategy classification: A falsificationist proposition. Judgment and Decision Making, 6, 814-820.

Newell, B. R., \& Fernandez, D. (2006). On the binary quality of recognition and the inconsequentially of further knowledge: Two critical tests of the recognition heuristic. Journal of Behavioral Decision Making, 19, 333-346.

Newell, B. R., Rakow, T., Weston, N. J., \& Shanks, D. R. (2004). Search strategies in decision making: The success of "success.". Journal of Behavioral Decision Making, 17, 117-137.

Newell, B. R., \& Shanks, D. R. (2003). Take the best or look at the rest? Factors influencing "one-reason" decision making. Journal of Experimental Psychology. Learning, Memory, and Cognition, 29, 53-65. doi:10.1037/0278-7393.29.1.53

Newell, B. R., \& Shanks, D. R. (2004). On the role of recognition in decision making. Journal of Experimental Psychology. Learning, Memory, and Cognition, 30, 923-935. doi:10.1037/0278-7393.30. 4.923

Oppenheimer, D. M. (2003). Not so fast! (and not so frugal!): Rethinking the recognition heuristic. Cognition, 90, B1-B9.

Pachur, T. (2011). The limited value of precise tests of the recognition heuristic. Judgment and Decision Making, 6, 413-422.

Pachur, T., Bröder, A., \& Marewski, J. (2008). The recognition heuristic in memory-based inference: Is recognition a noncompensatory cue? Journal of Behavioral Decision Making, $21,183-210$.

Pachur, T., \& Hertwig, R. (2006). On the psychology of the recognition heuristic: Retrieval primacy as a key determinant of its use. Journal of Experimental Psychology. Learning, Memory, and Cognition, 32, 983-1002.

Pachur, T., Mata, R., \& Schooler, L. J. (2009). Cognitive aging and the adaptive use of recognition in decision making. Psychology and Aging, 24, 901-915.

Pachur, T., \& Olsson, H. (2012). Type of learning task impacts performance and strategy selection in decision making. Cognitive Psychology, 65, 207-240.

Pachur, T., Todd, P. M., Gigerenzer, G., Schooler, L. J., \& Goldstein, D. G. (2011). The recognition heuristic: A review of theory and tests. Frontiers in Cognitive Science, 2(147), 1-14. doi:10.3389/fpsyg. 2011.00147

Payne, J. W. (1982). Contingent decision behavior. Psychological Bulletin, 92, 382-402.

Payne, J. W., Bettman, J. R., \& Johnson, E. J. (1988). Adaptive strategy selection in decision making. Journal of Experimental Psychology. Learning, Memory, and Cognition, 14, 534-552.

Payne, J. W., Bettman, J. R., \& Johnson, E. J. (1993). The adaptive decision maker. New York, NY: Cambridge University Press.

Platzer, C., \& Bröder, A. (2012). Most people do not ignore salient invalid cues in memory-based decisions. Psychonomic Bulletin \& Review, 19, 654-661. doi:10.3758/s13423-012-0248-4

Pohl, R. F. (2006). Empirical tests of the recognition heuristic. Journal of Behavioral Decision Making, 19, 251-271.

Pohl, R. F. (2011). On the use of recognition in inferential decision making: An overview of the debate. Judgment and Decision Making, 6, 423-438. 
Pohl, R. F., Erdfelder, E., Hilbig, B. E., Liebke, L., \& Stahlberg, D. (2013). Effort reduction after self-control depletion: The role of cognitive resources in use of simple heuristics. Journal of Cognitive Psychology, 25, 267-276.

Richter, T., \& Späth, P. (2006). Recognition is used as one cue among others in judgment and decision making. Journal of Experimental Psychology. Learning, Memory, and Cognition, 32, 150-162. doi: 10.1037/0278-7393.32.1.150

Rieskamp, J., \& Hoffrage, U. (2008). Inferences under time pressure: How opportunity costs affect strategy selection. Acta Psychologica, 127, 258-276.

Rouder, J. N., Speckman, P. L., Sun, D., Morey, R. D., \& Iverson, G. (2009). Bayesian $t$ tests for accepting and rejecting the null hypothesis. Psychonomic Bulletin \& Review, 16, 225-237. doi:10.3758/ PBR.16.2.225

Shah, A. K., \& Oppenheimer, D. M. (2007). Easy does it: The role of fluency in cue weighting. Judgment and Decision Making, 2, 371-379.
Shah, A. K., \& Oppenheimer, D. M. (2008). Heuristics made easy: An effort-reduction framework. Psychological Bulletin, 134, 207-222. doi:10.1037/0033-2909.134.2.207

Singmann, H., \& Kellen, D. (2013). MPTinR: Analysis of multinomial processing tree models in R. Behavior Research Methods, 45, 560-575.

Söllner, A., Bröder, A., \& Hilbig, B. E. (2013). Deliberation versus automaticity in decision making: Which presentation format features facilitate automatic decision making? Judgment and Decision Making, 8, 278-298.

Wagenmakers, E.-J. (2007). A practical solution to the pervasive problems of $p$ values. Psychonomic Bulletin \& Review, 14, 779-804. doi: 10.3758/BF03194105

Wasserman, L. (2000). Bayesian model selection and model averaging. Journal of Mathematical Psychology, 44, 92-107.

Weber, E. U., \& Johnson, E. J. (2009). Mindful judgment and decision making. Annual Review of Psychology, 60, 53-85. doi:10.1146/ annurev.psych.60.110707.163633 